\title{
Understanding the Role of Sensorimotor Beta Oscillations
}

\author{
Jacopo Barone ${ }^{\star \dagger}$ and Holly E. Rossiter ${ }^{\dagger}$ \\ Cardiff University Brain Research Imaging Centre, School of Psychology, Cardiff University, Cardiff, United Kingdom
}

Beta oscillations have been predominantly observed in sensorimotor cortices and basal ganglia structures and they are thought to be involved in somatosensory processing and motor control. Although beta activity is a distinct feature of healthy and pathological sensorimotor processing, the role of this rhythm is still under debate. Here we review recent findings about the role of beta oscillations during experimental manipulations (i.e., drugs and brain stimulation) and their alteration in aging and pathology. We show how beta changes when learning new motor skills and its potential to integrate sensory input with prior contextual knowledge. We conclude by discussing a novel methodological approach analyzing beta oscillations as a series of transient bursting events.

Keywords: beta rebound, beta desynchronization, beta bursts, brain oscillations, sensorimotor processing, functional role

\section{OPEN ACCESS}

Edited by:

Heiko J. Luhmann,

Johannes Gutenberg University

Mainz, Germany

Reviewed by:

Fritzie I. Arce-McShane,

University of Chicago, United States

Vladimir Litvak,

University College London,

United Kingdom

*Correspondence:

Jacopo Barone

BaroneJ@cardiff.ac.uk

${ }^{t}$ These authors have contributed equally to this work

Received: 19 January 2021 Accepted: 05 May 2021 Published: 31 May 2021

Citation: Barone $J$ and Rossiter HE (2021) Understanding the Role of Sensorimotor Beta Oscillations. Front. Syst. Neurosci. 15:655886. doi: 10.3389/fnsys.2021.655886

\section{INTRODUCTION}

Recordings of electrical signatures of the brain, via electroencephalography (EEG), magnetoencephalography (MEG), electrocorticography (ECoG), and local field potential (LFP), have consistently reported rhythmic patterns in neural activity. Brain rhythms, also known as oscillations, are linked with numerous cognitive functions and group into several oscillatory bands (Buzsáki, 2006). Beta oscillations $(\sim 13-30 \mathrm{~Hz})$ are commonly implicated in sensorimotor processing (Pfurtscheller and Lopes da Silva, 1999; Baker, 2007). These oscillations are established during stable postures and are decreased during active states, such as movement planning and execution (Engel and Fries, 2010; Kilavik et al., 2013). A decrease in the amplitude of beta oscillations across sensorimotor areas is seen just prior to and during movement execution. Conversely, an increase of beta amplitude above baseline levels is observed following movement cessation. We refer to these two phenomena as movement related beta decrease (MRBD) and post-movement beta rebound (PMBR), respectively (Figure 1A). The two principal sources of beta are sensorimotor cortex (Jensen et al., 2005; Roopun et al., 2006; Kramer et al., 2008; Yamawaki et al., 2008; Kopell et al., 2011) and basal ganglia (Figure 1C; Holgado et al., 2010; McCarthy et al., 2011; Tachibana et al., 2011; Mirzaei et al., 2017). There is debate as to whether they originate independently in each area or if they are an emergent property of the cortico-basal ganglia networks (Pavlides et al., 2015; Sherman et al., 2016; Reis et al., 2019).

The scope of this review is to report the most recent interpretations of the role of beta oscillations in the sensorimotor system. We will do so by giving details of how beta oscillations are affected by healthy aging (Rossiter et al., 2014b) and motor learning (Tan et al., 2014, 2016; Torrecillos et al., 2015; Haar and Faisal, 2020) as well as how they are altered in various conditions, most notably Parkinson's disease (PD) and stroke, characterized by severe motor symptoms (Brown, 2006; Rossiter et al., 2014a). We will also cover how beta oscillations can be manipulated experimentally 
using drugs and brain stimulation techniques. We will conclude by discussing how assessing the transient nature of beta oscillations could enrich our understating of its functional role (Feingold et al., 2015; Sherman et al., 2016; Shin et al., 2017).

\section{FUNCTIONAL ROLE OF BETA OSCILLATIONS}

Although several studies have extensively investigated beta oscillatory dynamics (Table 1) [for review, see Kilavik et al. (2013)], its functional role in the sensorimotor processing is still not fully understood. One source of interest lies in beta's tendency to fluctuate during movement. MRBD is present during spontaneous and triggered movements (Kilavik et al., 2013), while successful movement cancelation is associated with an increase in beta (Swann et al., 2009, 2012; Wagner et al., 2018; Jana et al., 2020). MRBD also occurs when no muscle contraction is required (i.e., motor imagery or action observation) and is rather insensitive to parameters like movement type or effector (Miller et al., 2010; Kilavik et al., 2013). These observations led Engel and Fries (2010) to propose a role for beta as an active process, which interferes with the encoding of incoming information while promoting the existing state-i.e., "statusquo"- of the system. Therefore, instead of being a proxy for the level of activity of the sensorimotor network, beta oscillations act as a top-down inhibitory rhythm during motor and cognitive tasks. Following the "status-quo" hypothesis, MRBD and PMBR could be interpreted as endogenous fluctuations of beta level during a motor set, with the former necessary for releasing the inhibition and allowing the initiation of a motor plan, while the latter preserves the existing motor states from internal and external sources of noise.

Post-movement beta rebound has also been interpreted more specifically as an indicator of movement outcome processing (Baker, 2007). Supporting evidence stems from findings showing PMBR is modulated by passive movements (Cassim et al., 2001; Alegre et al., 2002) and by kinematic errors (Tan et al., 2014). More recently, Tan et al. (2016) reported that the level of PMBR over the sensorimotor cortex serves as an index of confidence in the prediction of a motor outcome, also known as the forward model.

Together, these studies propose a role for sensorimotor beta oscillations encompassing multiple functions. We suggest several reasons why interpretations of beta's role are so varied.

First, beta involves several types of oscillations in distinct frequency bands (Kopell et al., 2011). PD studies show that oscillatory activity through the cortico-basal ganglia network is segregated into low $(14-20 \mathrm{~Hz})$ and high beta frequencies (>24 Hz) both in humans (López-Azcárate et al., 2010; Litvak et al., 2011) and rats (West et al., 2018). Furthermore, dopamine levels affect low and high beta rhythms differently (Brown et al., 2001; Priori et al., 2004; Marceglia et al., 2006). One theory proposes that low-beta has an "anti-kinetic" role (Brown, 2003; Chandrasekaran et al., 2019), while high-beta reflects attention and sensory cue anticipation (Saleh et al., 2010; Kilavik et al., 2014; Chandrasekaran et al., 2019).
TABLE 1 | Selective list of studies describing beta's functional role in the sensorimotor system.

\begin{tabular}{llll}
\hline \multicolumn{1}{c}{ Authors } & Locus & Function \\
\hline MRBD & Engel and Fries, 2010 & Cortex/Basal Ganglia & Motor plan initiation \\
& Jana et al., 2020 & Cortex & Action stopping \\
& Leventhal et al., 2012 & Basal Ganglia & Gating \\
& Little and Brown, 2014 & Basal Ganglia & Motor impairments \\
Shin et al., 2017 & Cortex & Attention, perception \\
& Stolk et al., 2019 & Cortex & Inhibition/excitation \\
Torrecillos et al., 2018 & Basal Ganglia & Motor control \\
PMBR & Cortex/Basal Ganglia & Movement outcome \\
& Cassim et al., 2001 & Cortex & Somatosensory processing \\
& Engel and Fries, 2010 & Cortex/Basal Ganglia & Motor states protection \\
& Feingold et al., 2015 & Basal Ganglia & Task performance, reward \\
Fry et al., 2016 & Cortex & Inhibition, motor control \\
Haar and Faisal, 2020 & Cortex & Motor learning \\
Tan et al., 2014, 2016 & Cortex & Predictive coding \\
Torrecillos et al., 2015 & Cortex & Error detection \\
\hline
\end{tabular}

The studies are split based on whether they focus more on MRBD (upper half) or PMBR (bottom half). MRBD: movement related beta decrease; PMBR: postmovement beta rebound.

Secondly, some studies have observed that MRBD and PMBR have a different spatial distribution (with MRBD localizing to postcentral gyrus and PMBR to precentral gyrus) and could represent independent events (Figure 1B; Jurkiewicz et al., 2006; Miller et al., 2007; Alegre et al., 2008; Gaetz et al., 2011; Muthukumaraswamy et al., 2013).

Finally, recent studies suggest that beta shows a burstlike activity, rather than being sustained over time. Therefore, theories of its functional role should integrate this transient nature and the potential implications for behavior (see section "Beta Oscillations as a Transient Rhythm").

\section{NATURAL VARIATION OF BETA OSCILLATIONS}

\section{Motor Learning}

A group of recent studies (Tan et al., 2014, 2016; Torrecillos et al., 2015; Alayrangues et al., 2019; Haar and Faisal, 2020) strengthen the link between beta oscillations and sensorimotor processes by testing for the role of beta in the context of motor learning. Motor learning can be defined as an improvement of motor skills through practice which is paralleled by long-lasting changes at the level of neural circuitry (Sanes and Donoghue, 2000; Muellbacher et al., 2002; Halsband and Lange, 2006). Motor learning paradigms involve goal-directed actions toward a target (reaching, pointing), while motor performance and/or sensory feedback (force fields, prisms) are experimentally manipulated [for review, see Shadmehr et al. (2010)].

In a visuomotor rotation task, Tan et al. $(2014,2016)$ found that larger PMBR amplitude indicates more confidence in the forward model and the maintenance of more stable motor output, while smaller PMBR indicates the need for adaptive changes driven by sensory feedback. Recently, Haar and Faisal (2020) explored PMBR dynamics during a real-world billiards task. Across the experiment, PMBR amplitude exhibited opposite modulations, with some participants showing a reduction with 


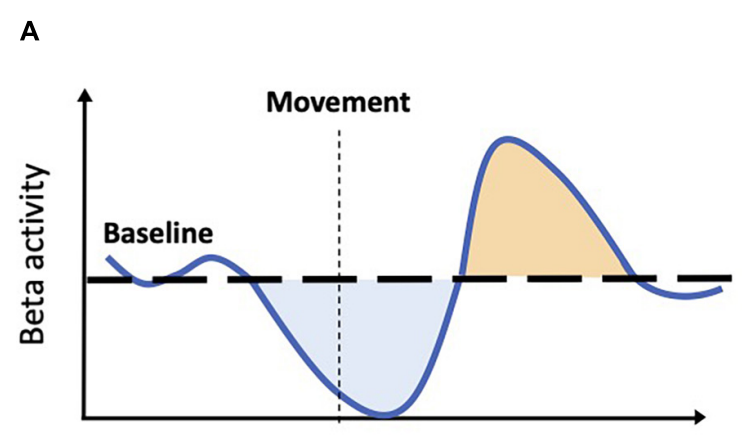

Time

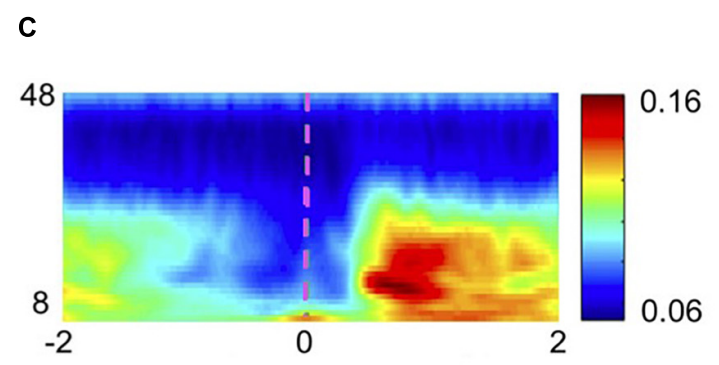

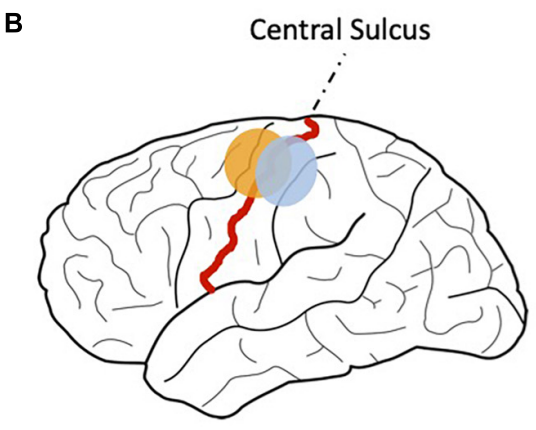

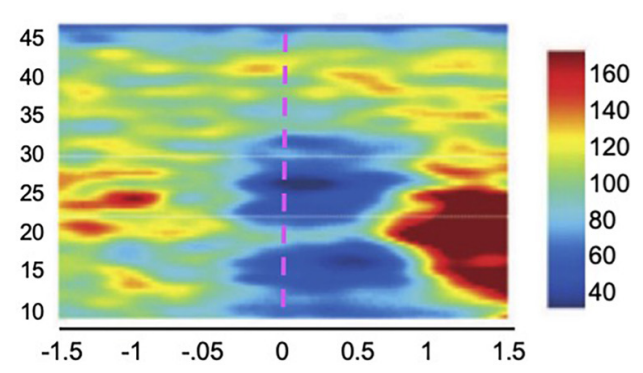

FIGURE 1 | MRBD and PMBR. (A) Schematic representation of sensorimotor beta activity. During a motor task beta activity drops below baseline (MRBD-light blue shaded area) just prior to and during movement execution. After movement ends, beta activity increases rapidly (PMBR-light orange shaded area) before slowly returning to baseline level. (B) Schematic representation of MRBD and PMBR spatial distribution. MRBD (light blue dot) is commonly localized to the postcentral gyrus whereas PMBR (light orange dot) is often localized to the precentral gyrus. (C) Time-frequency plots showing MRBD and PMBR in M1 (left-reproduced and adapted with permission from Little et al., 2019) and STN (right-reproduced and adapted with permission from Alegre et al., 2005). Both plots show oscillatory activity changes specific to the beta band (13-30 Hz) in the period prior to movement and following movement termination. The color scale indicates relative energy changes with respect to baseline level (blue colors indicate a decrease; red colors indicate an increase). The movement begins at time 0 (magenta dashed line). MRBD: movement related beta decrease; PMBR, post-movement beta rebound; M1: primary motor cortex; STN, subthalamic nucleus.

learning while others showed an increase. The authors speculated that participants may opt for distinct learning strategies to complete the task during real-world paradigms. Therefore, opposing PMBR dynamics could be interpreted as neural signatures of separate underlying learning mechanisms.

The link between PMBR and motor learning was also explored by Torrecillos et al. (2015). In a pointing task, the authors contrasted two types of reach errors: movement-execution errors that triggered adaptive mechanisms and errors that elicited no sensorimotor adaptation. PMBR amplitude was reduced after experiencing both kinds of errors, leading the authors to suggest a non-specific role for PMBR in error/mismatch detection. In a subsequent study, Alayrangues et al. (2019) contrasted bimanual reaching tasks with comparable motor kinematics but different action goals. Although each task required distinct sensorimotor remapping following a mechanical perturbation, PMBR modulation was comparable across tasks. This finding supports the notion that PMBR is related to salient errordetection mechanisms which act without triggering adaptive behavioral adjustments.

While beta's role in motor learning is still unresolved, new evidence suggest a complex relation of PMBR with outcome processing. Future studies need to clarify if the observed PMBR modulations during learning could be linked with changes in the upcoming motor output (in agreement with the "status-quo" hypothesis) or may reflect high-level sensory integration processes.

\section{Aging}

Motor performance is generally found to decline with age alongside concurrent changes in beta. Resting beta power has been shown to increase in older adults (Rossiter et al., 2014b; Heinrichs-Graham and Wilson, 2016; Espenhahn et al., 2019) as well as in youth (9-14 years) compared to adults (HeinrichsGraham and Wilson, 2016). An enhanced MRBD has been seen with increasing age both in older adults compared to younger adults (Heinrichs-Graham and Wilson, 2016; Provencher et al., 2016; Espenhahn et al., 2019) and in children compared to adults (Gaetz et al., 2010). MRBD was also found to reduce after motor learning (Gehringer et al., 2019). These studies suggest that concurrent increase in resting beta oscillations and MRBD may make the release of inhibition to initiate movement more difficult with aging.

From youth to adulthood, an increase in PMBR has been demonstrated (Gaetz et al., 2010) potentially linking to adults improved accuracy and ability to predict motor outcomes as found in Tan et al. (2016). There seems to be a link between changes in beta rebound and how people learn with age. Both 
Mary et al. (2015) and Moisello et al. (2015) found an enhanced PMBR due to learning in the contralateral sensorimotor cortex in younger adults which was not altered in older adults (Mary et al., 2015) or in PD patients (Moisello et al., 2015). Both authors suggest this may reflect plasticity in the younger adults allowing them to manipulate their beta levels during learning which was not observed in the older subjects or patients. Not only are resting beta oscillations stronger in older adults but it is harder for their PMBR to be altered in response to motor learning.

\section{Parkinson's Disease and Stroke}

Changes in sensorimotor beta oscillations have been observed in pathology such as PD and following stroke. Brown (2006) hypothesized that strong beta oscillations recorded from basal ganglia structures in PD patients may be antikinetic. Little and Brown (2014) did indeed find a strong correlation between beta and bradykinesia in PD patients. Contrastingly, a study by Heinrichs-Graham et al. (2014) demonstrated a reduced amplitude of beta oscillations in primary motor cortex of PD patients. They suggest this could be due to inhibitory drive originating from basal ganglia areas via the thalamus. When modulating dopamine levels in PD patients using levodopa, a suppression of beta power was seen in the subthalamic nucleus (Little et al., 2013) whereas an increase in beta power was seen in the motor cortex (Cao et al., 2020). These results fit with the theory that stronger beta amplitude, in this case in the basal ganglia, maintains the "status-quo" at the expense of voluntary movement although it highlights important differences between cortical and subcortical areas of the network.

Another neurotransmitter, GABA, is altered following stroke which affects levels of plasticity in the brain (Carmichael, 2012). Investigating beta following stroke can potentially give insight into these dynamics. Thibaut et al. (2017) found that higher resting beta power in the affected hemisphere of stroke patients was associated with poorer motor function whereas the reverse relationship was found in the unaffected hemisphere. Rossiter et al. (2014a) found a markedly diminished MRBD in stroke patients with motor impairment compared to healthy controls, and within the patient group, there was a correlation between MRBD and impairment, with reduced MRBD indicating greater level of motor impairment. Not only that but in Espenhahn et al. (2020), stroke patients' beta parameters were found to be modulated less following motor training than healthy controls. It is possible that stroke makes it more difficult for patients to modulate and suppress their beta oscillations in order to perform movements.

\section{EXPERIMENTAL MANIPULATION OF BETA OSCILLATIONS}

\section{Pharmacological Manipulation}

In order to assess the function of beta, it is possible to manipulate it experimentally. One such way of achieving this is through drugs. Changes to both resting state and motor task related activity in the beta band have been seen in response to drugs. At rest, giving benzodiazepines that increase the effect of GABA, such as lorazepam/diazepam, demonstrates a large increase in beta amplitude (Fingelkurts et al., 2004; Jensen et al., 2005; Hall et al., 2010). Studies assessing the effect of GABAergic drugs during simple finger movements demonstrated an increased resting beta power and an enhanced MRBD. PMBR was reduced by tiagabine (a non-specific GABA reuptake inhibitor) (Muthukumaraswamy et al., 2013) but was left unaltered by diazepam which is specific to GABA-A receptors (Hall et al., 2011) suggesting a potentially different mechanism behind MRBD and PMBR. Gaetz et al. (2011) demonstrated the link between GABA and beta oscillations using magnetic resonance spectroscopy and showed a positive correlation between levels of GABA in M1 and PMBR amplitude across individuals. These studies strongly suggest that the amplitude of beta oscillations is linked to levels of GABAergic inhibition in the brain.

\section{Brain Stimulation}

Brain stimulation is another method of manipulating beta in order to understand its underlying mechanisms in greater detail. In order to determine a causal role for beta oscillations, some studies have used transcranial alternating current stimulation (tACS) which stimulates superficial areas of cortex at specific frequencies in order to entrain neuronal firing. Pogosyan et al. (2009) were the first to show that beta frequency tACS over sensorimotor cortex slowed movement. This finding was backed up by Joundi et al. (2012) and Wach et al. (2013), with the latter showing that not only did beta tACS reduce the rate of force development, but gamma frequency tACS also increased it. Brain stimulation techniques offer a unique way of manipulating oscillations in the cortex in order to explore causal links with movement, however, positive findings are often hard to replicate (Héroux et al., 2017). Deep brain stimulation is a therapy in which areas of the basal ganglia are stimulated at high frequency. It can alleviate motor symptoms of disorders such as PD whilst simultaneously suppressing alpha and beta band activity across widespread areas including sensorimotor cortex and basal ganglia (Eusebio et al., 2011, 2012; Tinkhauser et al., 2017; Abbasi et al., 2018; Luoma et al., 2018). These studies point to a potential causal role of beta suppression in order to initiate movement.

\section{BETA OSCILLATIONS AS A TRANSIENT RHYTHM}

In previous reports, beta oscillations were typically considered as a repeated cycle of oscillatory activity sustained over time. A growing number of studies, however, are reconsidering beta oscillations as brief bursts of temporally localized activity (Feingold et al., 2015; Sherman et al., 2016; Shin et al., 2017; Tinkhauser et al., 2017; Torrecillos et al., 2018; Little et al., 2019). The idea of oscillations sustained across time stems from the standard analysis procedure of averaging oscillatory activity across many repeated trials (Jones, 2016). Sherman et al. (2016) analyzed source-localized human MEG data looking at spontaneous activity during rest in primary somatosensory cortex. In single trials, beta emerged transiently as a sudden increase in power typically lasting $<150 \mathrm{~ms}$, but when the 
same data was averaged over many trials, continuous oscillations appeared in the time-frequency spectrogram. Feingold et al. (2015) showed how beta-band events in LFPs occurred predominantly in brief bursts both in the motor-premotor cortex and in the striatum of monkeys performing self-timed movement tasks. Single trial analysis revealed that variations in averaged oscillatory power were expressed by variations in burst density of beta events. Thus, the authors suggest, beta synchronization and desynchronization reflect the probability of occurrence of a brief bursting event, rather than representing modulation of the strength of a sustained oscillation. Finally, Shin et al. (2017) showed that the rate of transient pre-stimulus beta events in the primary somatosensory cortex was the most consistent predictor of stimulus detection in humans and mice, while also being strongly correlated with average beta power. Taken together, cumulative evidence across brain areas, recording modalities and species support the notion of beta as a transient event. Trial averaged beta may conceal the functional importance of the underlying bursting activity, offering a less detailed interpretation of beta's role in the sensorimotor system. Whilst the averaged amplitude of beta power does seem to correlate with rate of beta events, implying that this new analysis may not contradict what has gone before, it will allow for more detailed exploration which may in turn help to consolidate the details of beta's role in sensorimotor integration.

\section{CONCLUSION}

Many of the studies in this review link to the idea that beta oscillations at rest ensure stability and the "status quo" of the motor system. Differently, studies focusing on PMBR highlight

\section{REFERENCES}

Abbasi, O., Hirschmann, J., Storzer, L., Özkurt, T. E., Elben, S., Vesper, J., et al. (2018). Unilateral deep brain stimulation suppresses alpha and beta oscillations in sensorimotor cortices. NeuroImage 174, 201-207. doi: 10.1016/j.neuroimage. 2018.03.026

Alayrangues, J., Torrecillos, F., Jahani, A., and Malfait, N. (2019). Error-related modulations of the sensorimotor post-movement and foreperiod beta-band activities arise from distinct neural substrates and do not reflect efferent signal processing. NeuroImage 184, 10-24. doi: 10.1016/j.neuroimage.2018.09.013

Alegre, M., Alonso-Frech, F., Rodríguez-Oroz, M. C., Guridi, J., Zamarbide, I., Valencia, M., et al. (2005). Movement-related changes in oscillatory activity in the human subthalamic nucleus: ipsilateral vs. contralateral movements. Eur. J. Neurosci. 22, 2315-2324. doi: 10.1111/j.1460-9568.2005.04409.x

Alegre, M., Alvarez-Gerriko, I., Valencia, M., Iriarte, J., and Artieda, J. (2008). Oscillatory changes related to the forced termination of a movement. Clin. Neurophysiol. 119, 290-300. doi: 10.1016/j.clinph.2007.10.017

Alegre, M., Labarga, A., Gurtubay, I. G., Iriarte, J., Malanda, A., and Artieda, J. (2002). Beta electroencephalograph changes during passive movements: sensory afferences contribute to beta event-related desynchronization in humans. Neurosci. Lett. 331, 29-32. doi: 10.1016/S0304-3940(02)00825-X

Baker, S. N. (2007). Oscillatory interactions between sensorimotor cortex and the periphery. Curr. Opin. Neurobiol. 17, 649-655. doi: 10.1016/j.conb.2008.01.007

Brown, P. (2003). Oscillatory nature of human basal ganglia activity: relationship to the pathophysiology of Parkinson's disease. Mov. Disord. Off. J. Mov. Disord. Soc. $18,357-363$. doi: $10.1002 /$ mds. 10358 a much more nuanced relationship with movement, potentially encoding features of motor learning and error-salience. Although beta's role is still not fully elucidated, it is unlikely to reflect pure sensory or motor processes. Instead, beta has become more broadly implicated in endogenous top-down processing and sensorimotor integration.

Future studies should work toward establishing a causal role of beta in the sensorimotor system. We described two distinct methods, pharmacology and brain stimulation, that can be effectively used to manipulate beta and infer causality. Furthermore, studies with patient groups with selective beta impairments, such as PD and stroke, can provide invaluable evidence of the physiological relevance of beta in motor control and motor learning. What should also be at the forefront in our minds is the richness of detail that may be gleaned with single trial analysis of beta events, which could return additional features to characterize and understand beta's role in the sensorimotor system.

\section{AUTHOR CONTRIBUTIONS}

Both authors contributed to the conception and design of the review, to the interpretation of the relevant literature, and to the writing and editing of the manuscript.

\section{FUNDING}

This work was funded by Cardiff University, School of Psychology (account no. AJ17106318). HR is supported by a Strategic Award from the Wellcome Trust (104943/Z/14/Z).

Brown, P. (2006). "Bad oscillations in Parkinson's disease," in Parkinson's Disease and Related Disorders, eds P. Riederer, H. Reichmann, M. B. H. Youdim, and M. Gerlach (Vienna: Springer), 27-30. doi: 10.1007/978-3-211-45295-0_6

Brown, P., Oliviero, A., Mazzone, P., Insola, A., Tonali, P., and Di Lazzaro, V. (2001). Dopamine dependency of oscillations between subthalamic nucleus and pallidum in Parkinson's disease. J. Neurosci. Off. J. Soc. Neurosci. 21, 1033-1038.

Buzsáki, G. (2006). Rhythms of the brain. New York, NY: Oxford University Press, doi: 10.1093/acprof:oso/9780195301069.001.0001

Cao, C., Li, D., Zhan, S., Zhang, C., Sun, B., and Litvak, V. (2020). L-dopa treatment increases oscillatory power in the motor cortex of Parkinson's disease patients. NeuroImage Clin. 26:102255. doi: 10.1016/j.nicl.2020.102255

Carmichael, S. T. (2012). Brain Excitability in Stroke: The Yin and Yang of Stroke Progression. Arch. Neurol. 69, 161-167. doi: 10.1001/archneurol.2011.1175

Cassim, F., Monaca, C., Szurhaj, W., Bourriez, J.-L., Defebvre, L., Derambure, P., et al. (2001). Does post-movement beta synchronization reflect an idling motor cortex? NeuroReport 12, 3859-3863.

Chandrasekaran, C., Bray, I. E., and Shenoy, K. V. (2019). Frequency Shifts and Depth Dependence of Premotor Beta Band Activity during Perceptual Decision-Making. J. Neurosci. Off. J. Soc. Neurosci. 39, 1420-1435. doi: 10.1523/ JNEUROSCI.1066-18.2018

Engel, A. K., and Fries, P. (2010). Beta-band oscillations-signalling the status quo? Curr. Opin. Neurobiol. 20, 156-165. doi: 10.1016/j.conb.2010.02.015

Espenhahn, S., Rossiter, H. E., van Wijk, B. C. M., Redman, N., Rondina, J. M., Diedrichsen, J., et al. (2020). Sensorimotor cortex beta oscillations reflect motor skill learning ability after stroke. Brain Commun. 2:fcaa161. doi: 10.1093/ braincomms/fcaal61 
Espenhahn, S., van Wijk, B. C. M., Rossiter, H. E., de Berker, A. O., Redman, N. D., Rondina, J., et al. (2019). Cortical beta oscillations are associated with motor performance following visuomotor learning. NeuroImage 195, 340-353. doi: 10.1016/j.neuroimage.2019.03.079

Eusebio, A., Cagnan, H., and Brown, P. (2012). Does suppression of oscillatory synchronisation mediate some of the therapeutic effects of DBS in patients with Parkinson's disease? Front. Integr. Neurosci. 6:47. doi: 10.3389/fnint.2012. 00047

Eusebio, A., Thevathasan, W., Gaynor, L. D., Pogosyan, A., Bye, E., Foltynie, T., et al. (2011). Deep brain stimulation can suppress pathological synchronisation in parkinsonian patients. J. Neurol. Neurosurg. Psychiatry 82, 569-573. doi: 10.1136/jnnp.2010.217489

Feingold, J., Gibson, D. J., DePasquale, B., and Graybiel, A. M. (2015). Bursts of beta oscillation differentiate postperformance activity in the striatum and motor cortex of monkeys performing movement tasks. Proc. Natl. Acad. Sci. 112, 13687-13692. doi: 10.1073/pnas.1517629112

Fingelkurts, A. A., Fingelkurts, A. A., Kivisaari, R., Pekkonen, E., Ilmoniemi, R. J., and Kähkönen, S. (2004). The interplay of lorazepam-induced brain oscillations: microstructural electromagnetic study. Clin. Neurophysiol. 115, 674-690. doi: 10.1016/j.clinph.2003.10.025

Fry, A., Mullinger, K. J., O’Neill, G. C., Barratt, E. L., Morris, P. G., Bauer, M., et al. (2016). Modulation of post-movement beta rebound by contraction force and rate of force development. Hum. Brain Mapp. 37, 2493-2511. doi: 10.1002/hbm. 23189

Gaetz, W., Edgar, J. C., Wang, D. J., and Roberts, T. P. L. (2011). Relating MEG measured motor cortical oscillations to resting $\gamma$-Aminobutyric acid (GABA) concentration. NeuroImage 55, 616-621. doi: 10.1016/j.neuroimage.2010. 12.077

Gaetz, W., MacDonald, M., Cheyne, D., and Snead, O. C. (2010). Neuromagnetic imaging of movement-related cortical oscillations in children and adults: Age predicts post-movement beta rebound. NeuroImage 51, 792-807. doi: 10.1016/ j.neuroimage.2010.01.077

Gehringer, J. E., Arpin, D. J., VerMaas, J. R., Trevarrow, M. P., Wilson, T. W., and Kurz, M. J. (2019). The Strength of the Movement-related Somatosensory Cortical Oscillations Differ between Adolescents and Adults. Sci. Rep. 9:18520. doi: 10.1038/s41598-019-55004-1

Haar, S., and Faisal, A. A. (2020). Brain Activity Reveals Multiple Motor-Learning Mechanisms in a Real-World Task. Front. Hum. Neurosci. 14:354. doi: 10.3389/ fnhum.2020.00354

Hall, S. D., Barnes, G. R., Furlong, P. L., Seri, S., and Hillebrand, A. (2010). Neuronal network pharmacodynamics of GABAergic modulation in the human cortex determined using pharmaco-magnetoencephalography. Hum. Brain Mapp. 31, 581-594. doi: 10.1002/hbm.20889

Hall, S. D., Stanford, I. M., Yamawaki, N., McAllister, C. J., Rönnqvist, K. C., Woodhall, G. L., et al. (2011). The role of GABAergic modulation in motor function related neuronal network activity. NeuroImage 56, 1506-1510. doi: 10.1016/j.neuroimage.2011.02.025

Halsband, U., and Lange, R. K. (2006). Motor learning in man: a review of functional and clinical studies. J. Physiol. Paris 99, 414-424. doi: 10.1016/j. jphysparis.2006.03.007

Heinrichs-Graham, E., and Wilson, T. W. (2016). Is an absolute level of cortical beta suppression required for proper movement? Magnetoencephalographic evidence from healthy aging. NeuroImage 134, 514-521. doi: 10.1016/j. neuroimage.2016.04.032

Heinrichs-Graham, E., Kurz, M. J., Becker, K. M., Santamaria, P. M., Gendelman, H. E., and Wilson, T. W. (2014). Hypersynchrony despite pathologically reduced beta oscillations in patients with Parkinson's disease: a pharmacomagnetoencephalography study. J. Neurophysiol. 112, 1739-1747. doi: 10.1152/ jn.00383.2014

Héroux, M. E., Loo, C. K., Taylor, J. L., and Gandevia, S. C. (2017). Questionable science and reproducibility in electrical brain stimulation research. PLoS One 12:e0175635. doi: 10.1371/journal.pone.0175635

Holgado, A. J. N., Terry, J. R., and Bogacz, R. (2010). Conditions for the Generation of Beta Oscillations in the Subthalamic Nucleus-Globus Pallidus Network. J. Neurosci. 30, 12340-12352. doi: 10.1523/JNEUROSCI.0817-10.2010

Jana, S., Hannah, R., Muralidharan, V., and Aron, A. R. (2020). Temporal cascade of frontal, motor and muscle processes underlying human action-stopping. eLife 9:e50371. doi: 10.7554/eLife.50371
Jensen, O., Goel, P., Kopell, N., Pohja, M., Hari, R., and Ermentrout, B. (2005). On the human sensorimotor-cortex beta rhythm: Sources and modeling. NeuroImage 26, 347-355. doi: 10.1016/j.neuroimage.2005.02.008

Jones, S. R. (2016). When brain rhythms aren't 'rhythmic': implication for their mechanisms and meaning. Curr. Opin. Neurobiol. 40, 72-80. doi: 10.1016/j. conb.2016.06.010

Joundi, R. A., Jenkinson, N., Brittain, J.-S., Aziz, T. Z., and Brown, P. (2012). Driving Oscillatory Activity in the Human Cortex Enhances Motor Performance. Curr. Biol. 22, 403-407. doi: 10.1016/j.cub.2012.01.024

Jurkiewicz, M. T., Gaetz, W. C., Bostan, A. C., and Cheyne, D. (2006). Post-movement beta rebound is generated in motor cortex: Evidence from neuromagnetic recordings. NeuroImage 32, 1281-1289. doi: 10.1016/j. neuroimage.2006.06.005

Kilavik, B. E., Confais, J., and Riehle, A. (2014). Signs of timing in motor cortex during movement preparation and cue anticipation. Adv. Exp. Med. Biol. 829, 121-142. doi: 10.1007/978-1-4939-1782-2_7

Kilavik, B. E., Zaepffel, M., Brovelli, A., MacKay, W. A., and Riehle, A. (2013). The ups and downs of beta oscillations in sensorimotor cortex. Exp. Neurol. 245, 15-26. doi: 10.1016/j.expneurol.2012.09.014

Kopell, N., Whittington, M. A., and Kramer, M. A. (2011). Neuronal assembly dynamics in the betal frequency range permits short-term memory. Proc. Natl. Acad. Sci. 108, 3779-3784. doi: 10.1073/pnas.1019676108

Kramer, M. A., Roopun, A. K., Carracedo, L. M., Traub, R. D., Whittington, M. A., and Kopell, N. J. (2008). Rhythm Generation through Period Concatenation in Rat Somatosensory Cortex. PLoS Comput. Biol. 4:e1000169. doi: 10.1371/ journal.pcbi.1000169

Leventhal, D. K., Gage, G. J., Schmidt, R., Pettibone, J. R., Case, A. C., and Berke, J. D. (2012). Basal Ganglia Beta Oscillations Accompany Cue Utilization. Neuron 73, 523-536. doi: 10.1016/j.neuron.2011.11.032

Little, S., and Brown, P. (2014). The functional role of beta oscillations in Parkinson's disease. Parkinsonism Relat. Disord. 20, S44-S48. doi: 10.1016/ S1353-8020(13)70013-0

Little, S., Bonaiuto, J., Barnes, G., and Bestmann, S. (2019). Human motor cortical beta bursts relate to movement planning and response errors. PLoS Biol. 17:3000479. doi: 10.1371/journal.pbio.3000479

Little, S., Tan, H., Anzak, A., Pogosyan, A., Kühn, A., and Brown, P. (2013). Bilateral Functional Connectivity of the Basal Ganglia in Patients with Parkinson's Disease and Its Modulation by Dopaminergic Treatment. PLoS One 8:e82762. doi: 10.1371/journal.pone.0082762

Litvak, V., Jha, A., Eusebio, A., Oostenveld, R., Foltynie, T., Limousin, P., et al (2011). Resting oscillatory cortico-subthalamic connectivity in patients with Parkinson's disease. Brain J. Neurol. 134, 359-374. doi: 10.1093/brain/awq332

López-Azcárate, J., Tainta, M., Rodríguez-Oroz, M. C., Valencia, M., González, R. Guridi, J., et al. (2010). Coupling between beta and high-frequency activity in the human subthalamic nucleus may be a pathophysiological mechanism in Parkinson's disease. J. Neurosci. Off. J. Soc. Neurosci. 30, 6667-6677. doi: 10.1523/JNEUROSCI.5459-09.2010

Luoma, J., Pekkonen, E., Airaksinen, K., Helle, L., Nurminen, J., Taulu, S., et al. (2018). Spontaneous sensorimotor cortical activity is suppressed by deep brain stimulation in patients with advanced Parkinson's disease. Neurosci. Lett. 683, 48-53. doi: 10.1016/j.neulet.2018.06.041

Marceglia, S., Foffani, G., Bianchi, A. M., Baselli, G., Tamma, F., Egidi, M., et al. (2006). Dopamine-dependent non-linear correlation between subthalamic rhythms in Parkinson's disease. J. Physiol. 571, 579-591. doi: 10.1113/jphysiol. 2005.100271

Mary, A., Bourguignon, M., Wens, V., Op, de Beeck, M., Leproult, R., et al. (2015). Aging reduces experience-induced sensorimotor plasticity. A magnetoencephalographic study. NeuroImage 104, 59-68. doi: 10.1016/j. neuroimage.2014.10.010

McCarthy, M. M., Moore-Kochlacs, C., Gu, X., Boyden, E. S., Han, X., and Kopell, N. (2011). Striatal origin of the pathologic beta oscillations in Parkinson's disease. Proc. Natl. Acad. Sci. 108, 11620-11625. doi: 10.1073/pnas.1107748108

Miller, K. J., Leuthardt, E. C., Schalk, G., Rao, R. P. N., Anderson, N. R., Moran, D. W., et al. (2007). Spectral Changes in Cortical Surface Potentials during Motor Movement. J. Neurosci. 27, 2424-2432. doi: 10.1523/JNEUROSCI.388606.2007

Miller, K. J., Schalk, G., Fetz, E. E., Nijs, M., den, Ojemann, J. G., et al. (2010). Cortical activity during motor execution, motor imagery, and imagery-based 
online feedback. Proc. Natl. Acad. Sci. 107, 4430-4435. doi: 10.1073/pnas. 0913697107

Mirzaei, A., Kumar, A., Leventhal, D., Mallet, N., Aertsen, A., Berke, J., et al. (2017). Sensorimotor Processing in the Basal Ganglia Leads to Transient Beta Oscillations during Behavior. J. Neurosci. 37, 11220-11232. doi: 10.1523/ JNEUROSCI.1289-17.2017

Moisello, C., Blanco, D., Lin, J., Panday, P., Kelly, S. P., Quartarone, A., et al. (2015). Practice changes beta power at rest and its modulation during movement in healthy subjects but not in patients with Parkinson's disease. Brain Behav. 5:e00374. doi: 10.1002/brb3.374

Muellbacher, W., Ziemann, U., Wissel, J., Dang, N., Kofler, M., Facchini, S., et al. (2002). Early consolidation in human primary motor cortex. Nature 415, 640-644. doi: 10.1038/nature712

Muthukumaraswamy, S. D., Myers, J. F. M., Wilson, S. J., Nutt, D. J., LingfordHughes, A., Singh, K. D., et al. (2013). The effects of elevated endogenous GABA levels on movement-related network oscillations. NeuroImage 66, 36-41. doi: 10.1016/j.neuroimage.2012.10.054

Pavlides, A., Hogan, S. J., and Bogacz, R. (2015). Computational Models Describing Possible Mechanisms for Generation of Excessive Beta Oscillations in Parkinson's Disease. PLoS Comput. Biol. 11:e1004609. doi: 10.1371/journal. pcbi.1004609

Pfurtscheller, G., and Lopes da Silva, F. H. (1999). Event-related EEG/MEG synchronization and desynchronization: basic principles. Clin. Neurophysiol. 110, 1842-1857. doi: 10.1016/S1388-2457(99)00141-8

Pogosyan, A., Gaynor, L. D., Eusebio, A., and Brown, P. (2009). Boosting cortical activity at Beta-band frequencies slows movement in humans. Curr. Biol. CB 19, 1637-1641. doi: 10.1016/j.cub.2009.07.074

Priori, A., Foffani, G., Pesenti, A., Tamma, F., Bianchi, A. M., Pellegrini, M., et al. (2004). Rhythm-specific pharmacological modulation of subthalamic activity in Parkinson's disease. Exp. Neurol. 189, 369-379. doi: 10.1016/j.expneurol.2004. 06.001

Provencher, D., Hennebelle, M., Cunnane, S. C., Bérubé-Lauzière, Y., and Whittingstall, K. (2016). Cortical Thinning in Healthy Aging Correlates with Larger Motor-Evoked EEG Desynchronization. Front. Aging Neurosci. 8:63. doi: 10.3389/fnagi.2016.00063

Reis, C., Sharott, A., Magill, P. J., van Wijk, B. C. M., Parr, T., Zeidman, P., et al. (2019). Thalamocortical dynamics underlying spontaneous transitions in beta power in Parkinsonism. Neuroimage 193, 103-114. doi: 10.1016/j.neuroimage. 2019.03.009

Roopun, A. K., Middleton, S. J., Cunningham, M. O., LeBeau, F. E. N., Bibbig, A., Whittington, M. A., et al. (2006). A beta2-frequency $(20-30 \mathrm{~Hz})$ oscillation in nonsynaptic networks of somatosensory cortex. Proc. Natl. Acad. Sci. 103, 15646-15650. doi: 10.1073/pnas.0607443103

Rossiter, H. E., Boudrias, M.-H., and Ward, N. S. (2014a). Do movement-related beta oscillations change after stroke? J. Neurophysiol. 112, 2053-2058. doi: 10.1152/jn.00345.2014

Rossiter, H. E., Davis, E. M., Clark, E. V., Boudrias, M.-H., and Ward, N. S. (2014b). Beta oscillations reflect changes in motor cortex inhibition in healthy ageing. NeuroImage 91, 360-365. doi: 10.1016/j.neuroimage.2014.01.012

Saleh, M., Reimer, J., Penn, R., Ojakangas, C. L., and Hatsopoulos, N. G. (2010). Fast and Slow Oscillations in Human Primary Motor Cortex Predict Oncoming Behaviorally Relevant Cues. Neuron 65, 461-471. doi: 10.1016/j.neuron.2010. 02.001

Sanes, J. N., and Donoghue, J. P. (2000). Plasticity and primary motor cortex. Annu. Rev. Neurosci. 23, 393-415. doi: 10.1146/annurev.neuro.23.1.393

Shadmehr, R., Smith, M. A., and Krakauer, J. W. (2010). Error Correction, Sensory Prediction, and Adaptation in Motor Control. Annu. Rev. Neurosci. 33, 89-108. doi: 10.1146/annurev-neuro-060909-153135

Sherman, M. A., Lee, S., Law, R., Haegens, S., Thorn, C. A., Hämäläinen, M. S., et al. (2016). Neural mechanisms of transient neocortical beta rhythms: Converging evidence from humans, computational modeling, monkeys, and mice. Proc. Natl. Acad. Sci. 113, E4885-E4894. doi: 10.1073/pnas.1604135113

Shin, H., Law, R., Tsutsui, S., Moore, C. I., and Jones, S. R. (2017). The rate of transient beta frequency events predicts behavior across tasks and species. eLife 6:e29086. doi: 10.7554/eLife.29086

Stolk, A., Brinkman, L., Vansteensel, M. J., Aarnoutse, E., Leijten, F. S., Dijkerman, C. H., et al. (2019). Electrocorticographic dissociation of alpha and beta rhythmic activity in the human sensorimotor system. eLife 8:e48065. doi: 10 . 7554/eLife.48065

Swann, N. C., Cai, W., Conner, C. R., Pieters, T. A., Claffey, M. P., George, J. S., et al. (2012). Roles for the pre-supplementary motor area and the right inferior frontal gyrus in stopping action: Electrophysiological responses and functional and structural connectivity. NeuroImage 59, 2860-2870. doi: 10. 1016/j.neuroimage.2011.09.049

Swann, N., Tandon, N., Canolty, R., Ellmore, T. M., McEvoy, L. K., Dreyer, S., et al. (2009). Intracranial EEG Reveals a Time- and Frequency-Specific Role for the Right Inferior Frontal Gyrus and Primary Motor Cortex in Stopping Initiated Responses. J. Neurosci. 29, 12675-12685. doi: 10.1523/JNEUROSCI.3359-09. 2009

Tachibana, Y., Iwamuro, H., Kita, H., Takada, M., and Nambu, A. (2011). Subthalamo-pallidal interactions underlying parkinsonian neuronal oscillations in the primate basal ganglia. Eur. J. Neurosci. 34, 1470-1484. doi: 10.1111/j.1460-9568.2011.07865.x

Tan, H., Jenkinson, N., and Brown, P. (2014). Dynamic neural correlates of motor error monitoring and adaptation during trial-to-trial learning. J. Neurosci. Off. J. Soc. Neurosci. 34, 5678-5688. doi: 10.1523/JNEUROSCI.4739-13.2014

Tan, H., Wade, C., and Brown, P. (2016). Post-Movement Beta Activity in Sensorimotor Cortex Indexes Confidence in the Estimations from Internal Models. J. Neurosci. 36, 1516-1528. doi: 10.1523/JNEUROSCI.320415.2016

Thibaut, A., Simis, M., Battistella, L. R., Fanciullacci, C., Bertolucci, F., HuertaGutierrez, R., et al. (2017). Using Brain Oscillations and Corticospinal Excitability to Understand and Predict Post-Stroke Motor Function. Front. Neurol. 8:187. doi: 10.3389/fneur.2017.00187

Tinkhauser, G., Pogosyan, A., Little, S., Beudel, M., Herz, D. M., Tan, H., et al. (2017). The modulatory effect of adaptive deep brain stimulation on beta bursts in Parkinson's disease. Brain 140, 1053-1067. doi: 10.1093/brain/ awx010

Torrecillos, F., Alayrangues, J., Kilavik, B. E., and Malfait, N. (2015). Distinct Modulations in Sensorimotor Postmovement and Foreperiod $\beta$-Band Activities Related to Error Salience Processing and Sensorimotor Adaptation. J. Neurosci. 35, 12753-12765. doi: 10.1523/JNEUROSCI.1090-15.2015

Torrecillos, F., Tinkhauser, G., Fischer, P., Green, A. L., Aziz, T. Z., Foltynie, T., et al. (2018). Modulation of Beta Bursts in the Subthalamic Nucleus Predicts Motor Performance. J. Neurosci. 38, 8905-8917. doi: 10.1523/JNEUROSCI. 1314-18.2018

Wach, C., Krause, V., Moliadze, V., Paulus, W., Schnitzler, A., and Pollok, B. (2013). Effects of $10 \mathrm{~Hz}$ and $20 \mathrm{~Hz}$ transcranial alternating current stimulation (tACS) on motor functions and motor cortical excitability. Behav. Brain Res. 241, 1-6. doi: 10.1016/j.bbr.2012.11.038

Wagner, J., Wessel, J. R., Ghahremani, A., and Aron, A. R. (2018). Establishing a right frontal beta signature for stopping action in scalp EEG: implications for testing inhibitory control in other task contexts. J. Cogn. Neurosci. 30, 107-118. doi: 10.1162/jocn_a_01183

West, T. O., Berthouze, L., Halliday, D. M., Litvak, V., Sharott, A., Magill, P. J., et al. (2018). Propagation of beta/gamma rhythms in the cortico-basal ganglia circuits of the parkinsonian rat. J. Neurophysiol. 119, 1608-1628. doi: 10.1152/ jn.00629.2017

Yamawaki, N., Stanford, I. M., Hall, S. D., and Woodhall, G. L. (2008). Pharmacologically induced and stimulus evoked rhythmic neuronal oscillatory activity in the primary motor cortex in vitro. Neuroscience 151, 386-395. doi: 10.1016/j.neuroscience.2007.10.021

Conflict of Interest: The authors declare that the research was conducted in the absence of any commercial or financial relationships that could be construed as a potential conflict of interest.

Copyright (c) 2021 Barone and Rossiter. This is an open-access article distributed under the terms of the Creative Commons Attribution License (CC BY). The use, distribution or reproduction in other forums is permitted, provided the original author(s) and the copyright owner(s) are credited and that the original publication in this journal is cited, in accordance with accepted academic practice. No use, distribution or reproduction is permitted which does not comply with these terms. 